\title{
The Microstructure of Pharmaceutical Materials Revealed by Scanning Electron Diffraction
}

\author{
Duncan N. Johnstone ${ }^{1}$ and Paul A. Midgley ${ }^{1}$ \\ 1. Department of Materials Science and Metallurgy, University of Cambridge, 27 Charles Babbage Road, \\ Cambridge, UK.
}

The microstructure of pharmaceutical materials influences important physical properties [1] such as compaction behavior, dissolution rate, bioavailability, hygroscopicity, dehydration, chemical stability and physical stability. However, few techniques are capable of directly revealing the underlying microstructure and associated defects. Transmission electron microscopy (TEM) provides an obvious means to image and characterize microstructure and defects. Whilst previous TEM studies of pharmaceutical materials have been insightful [2] the level of detail attained has been necessarily limited because of the sensitivity of the sample to damage by electron irradiation. Scanning electron diffraction (SED) provides a new paradigm in which the data required for detailed analysis is acquired rapidly and before the specimen is destroyed. Insight is gleaned post-facto through the application of versatile computational analysis enabling maximal information to be extracted from the data.

SED involves scanning a convergent electron probe across the specimen and recording an electron diffraction pattern at each probe position. Typically, $\sim 10^{5}$ diffraction patterns are recorded yielding data rich in structural information with a spatial resolution of $\sim 1-10 \mathrm{~nm}$ across areas of $\sim 0.1-1 \mu \mathrm{m}$. In this work, SED was applied to acetaminophen crystals, as purchased in powder form from Sigma-Aldrich. This provides a proof-of-principle demonstration in an extensively studied system where interest remains in understanding defects and controlling mechanical deformation [3]. SED was performed using a Philips CM300 FEG-TEM retrofitted with a NanoMEGAS ASTAR system to control the beam scan and simultaneous acquisition of electron diffraction patterns. The instrument was operated at $300 \mathrm{keV}$ with the sample cooled to liquid nitrogen temperatures to minimize beam damage. A scan step size of 20 $\mathrm{nm}$ was chosen as a compromise between spatial resolution and field of view and a pixel dwell time of $10 \mathrm{~ms}$ further minimized beam damage.

The 4D-SED data may be analyzed in many ways to provide insight in both real and reciprocal space. Firstly, the diffracting vectors present in each diffraction pattern can be considered directly to assess the crystal phases present. Here it was found that the crystal adopts the monoclinic polymorph as thermodynamically expected. In Figure 1, the average diffraction pattern from the region of interest reveals that the crystal is rotating slightly over the field of view, exciting a series of reflections differing by a [001]* g-vector. 'Virtual' dark-field (VDF) images can be formed by plotting the diffracted intensity at a particular g-vector (or set of vectors) as function of probe position. The morphology of the crystals is revealed along with local variations in the diffracted intensity, indicative of distortions to the crystal structure. Two such examples are shown in Figure 1 suggesting a network of dislocations in the top of the region. Such contrast can be used to assess the character of the particular defects. More advanced computational analysis may also be employed, including methods of unsupervised machine learning. Here, non-negative matrix factorization (NMF), was applied to learn the 'component' diffraction patterns in the data as well as the real space 'loading' of these components. The relationship between the crystal domains, revealed by NMF, can be assessed based on the learnt component diffraction patterns, shown in Figure 2. 
In conclusion, SED is presented as a versatile approach to revealing defect structures in pharmaceutical crystals when coupled with powerful post-facto computation analysis. This approach offers the potential to develop detailed understanding of microstructure-property relationships in pharmaceuticals [6] [4].

\section{References:}

[1] W. Jones and M.D. Eddleston in "Disordered Pharmaceutical Materials", (Wiley), p. 85.

[2] M.D. Eddleston et al, Journal of Pharmaceutical Sciences, 99, (2010), p.4072.

[3] S. Finnie et al, Pharmaceutical Research, 18, (2001), p.674.

[4] The authors acknowledge funding from the University of Cambridge, ERC grant 291522-

3DIMAGE, European Commission grant 312483-ESTEEM2 and the EPSRC NanoDTC EP/L015978/1.
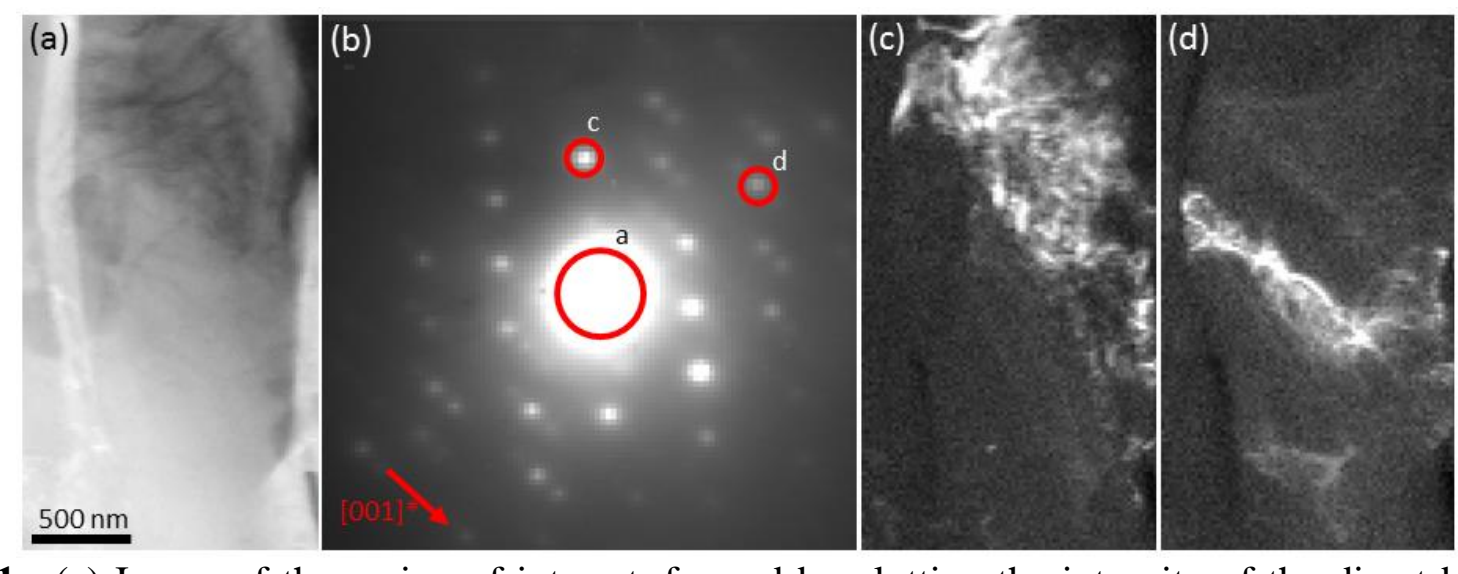

Figure 1. (a) Image of the region of interest, formed by plotting the intensity of the direct beam as a function of probe position. (b) Summed diffraction from the region of interest with integration windows used to form $(\mathrm{c}, \mathrm{d})$ the virtual dark field images shown along with the g-vector [001]*. Contrast in the VDF images reveals what is most likely a network of dislocations.
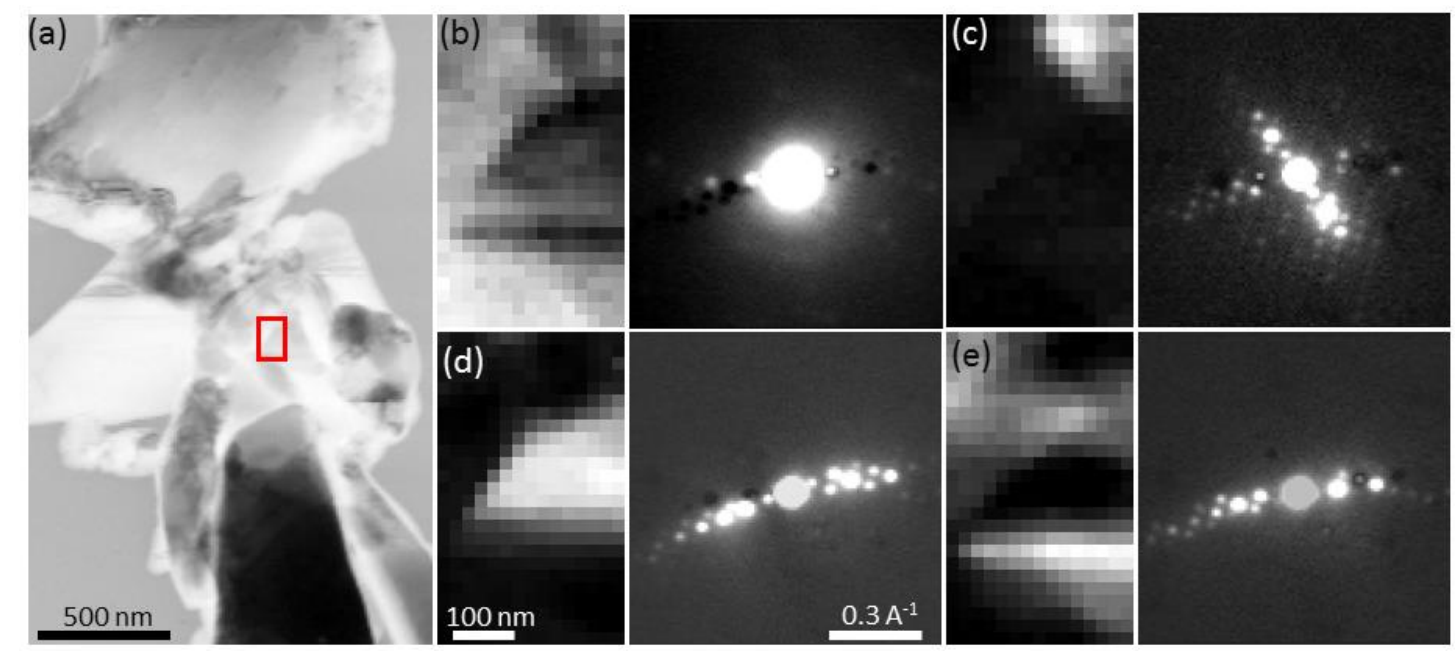

Figure 2. (a) Virtual image formed by plotting intensity in the direct beam, providing an overview of a second region of interest. The dark object at the bottom of the region is thick material and a number of aggregated crystals are evident. (b-e) Non-negative matrix factorization yields the real space loading of particular component diffraction patterns. These component patterns can be used to reveal the relationship between neighboring crystals. 